\title{
Invariant Image-Based Currency Denomination Recognition Using Local Entropy and Range Filters
}

\author{
Hafeez Anwar 1,2, Farman Ullah ${ }^{2}$, Asif Iqbal ${ }^{3}$, Anees Ul Hasnain ${ }^{4}$, Ata Ur Rehman ${ }^{2}$, \\ Peter Bell ${ }^{1, * \mathbb{D}}$ and Daehan Kwak ${ }^{5, * \text { (D) }}$ \\ 1 Interdisciplinary Center for Digital Humanities and Social Sciences, Friedrich-Alexander-Universität \\ Erlangen-Nürnberg, 91052 Erlangen, Germany; hafeez.anwar@fau.de \\ 2 Department of Electrical \& Computer Engineering, COMSATS University Islamabad-Attock Campus, \\ Attock 43600, Pakistan; farmankttk@cuiatk.edu.pk (F.U.); dr.ataurrehman@cuiatk.edu.pk (A.U.R.) \\ 3 Department of Information and Communication Engineering, Inha University, Incheon 22212, Korea; \\ asifsoul@inha.ac.kr \\ 4 EPAS Engineering, Topi 23460, Pakistan; anees@epas.com.pk \\ 5 Department of Computer Science, Kean University, Union, NJ 07083, USA \\ * Correspondence: peter.bell@fau.de (P.B.); dkwak@kean.edu (D.K.)
}

Received: 20 September 2019; Accepted: 2 November 2019; Published: 6 November 2019

\begin{abstract}
We perform image-based denomination recognition of the Pakistani currency notes. There are a total of seven different denominations in the current series of Pakistani notes. Apart from color and texture, these notes differ from one another mainly due to their aspect ratios. Our aim is to exploit this single feature to attain an image-based recognition that is invariant to the most common image variations found in currency notes images. Among others, the most notable image variations are caused by the difference in positions and in-plane orientations of the currency notes in images. While most of the proposed methods for currency denomination recognition only focus on attaining higher recognition rates, our aim is more complex, i.e., attaining a high recognition rate in the presence of image variations. Since, the aspect ratio of a currency note is invariant to such differences, an image-based recognition of currency notes based on aspect ratio is more likely to be translation- and rotation-invariant. Therefore, we adapt a two step procedure that first extracts a currency note from the homogeneous image background via local entropy and range filters. Then, the aspect ratio of the extracted currency note is calculated to determine its denomination. To validate our proposed method, we gathered a new dataset with the largest and most diverse collection of Pakistani currency notes, where each image contains either a single or multiple notes at arbitrary positions and orientations. We attain an overall average recognition rate of $99 \%$ which is very encouraging for our method, which relies on a single feature and is suited for real-time applications. Consequently, the method may be extended to other international and historical currencies, which makes it suitable for business and digital humanities applications.
\end{abstract}

Keywords: image entropy; image processing; image segmentation

\section{Introduction}

In this paper, we propose an image-based framework for the denomination recognition of Pakistani currency notes of the 2005 series. Such systems are important as they are used at several situations such as automatic vending machines and supporting visually impaired people [1] to identify the denomination of a given currency note. Consequently, such image-based denomination recognition of currency notes has become an active area of research where the proposed solutions can be divided into two main groups. The first group of methods [2] deals with currency images that are acquired 
with scanners. Such methods are more suitable for applications like automatic teller machines (ATM). However, the most common variations that are found in scanned images are the ones induced by the variations in currency notes orientation, scale and position. The second group of methods deals with currency note images that are taken with a camera in a cluttered environment. Such a system installed on a smart phone can be helpful for visually impaired people in their daily life. However, these methods face challenges due to the image variations caused by non-uniform illumination, background clutter, and partial occlusions.

The proposed solutions are broadly divided into three different groups, where the first group uses the so-called local features matching such as scale-invariant feature transform (SIFT) [3]. The second group makes use of supervised machine learning algorithms such as artificial neural networks (ANNs) [4]. Lastly, the third group of methods uses pure image processing techniques such as template matching [5].

We take a different approach to the problem of image-based denomination recognition of scanned currency notes. We are interested in utilizing simple image filtering, rather than using a complex handcrafted local image descriptor or a complicated machine learning algorithm such as a convolutional neural network (CNN). In addition to simplicity, such filtering is also invariant to the most commonly found variations in the currency notes images. These image variations are not explicitly identified and dealt with in any of the previously proposed methods. Figure 1 depicts the variations that are found in the currency note images. The orientation differences found among the currency notes of any given denomination can cause variations in their images. This makes images of the same currency note look different from one another. This can clearly be observed in the third column of Figure 1. Similarly, the variations caused by position and scale differences of the currency notes induce variations in their images which are depicted in the first and second columns of Figure 1. Lastly, the image variations can also be caused by multiple currency notes in a single image as shown in fourth column of Figure 1. Apart from image variations, the recognition rate is also likely to be affected by the variations caused by the condition of currency notes themselves. The standard aspect ratios of currency notes are based on the freshly printed and unused notes as shown in Table 1. However, the excessive and rough use of the currency notes causes wear and tear, due to which, their boundaries become irregular. Since, we propose to use the aspect ratio as a single feature for recognizing these notes, such irregularities in the boundaries induce variations in the aspect ratios as well. This is demonstrated in Figure 2 where currency notes of multiple frequently used denominations are shown. It can be observed that the aspect ratios of currency notes belonging to a single denomination vary from one another. However, it should be noted that the measurements are based on our proposed image-based method. To summarize, in order to achieve an accurate denomination recognition of currency notes, it is important to address these variations. This has already been proved in other object categories such as ancient coins [6], and butterflies and fish [7], where image variations caused by changes in object orientation, scale, and position are very common.

Table 1. The Pakistani currency notes of the 2005 Series along with their width, height, and aspect ratios. The aspect ratio is calculated as (height/width) $\times 10,000$.

\begin{tabular}{|c|c|c|c|c|c|c|c|}
\hline & & & & & & & \\
\hline Height & 65 & 65 & 65 & 65 & 65 & 65 & 65 \\
\hline Width & 115 & 123 & 131 & 139 & 147 & 155 & 163 \\
\hline Aspect ratio & 5652 & 5285 & 4962 & 4676 & 4422 & 4194 & 3988 \\
\hline
\end{tabular}




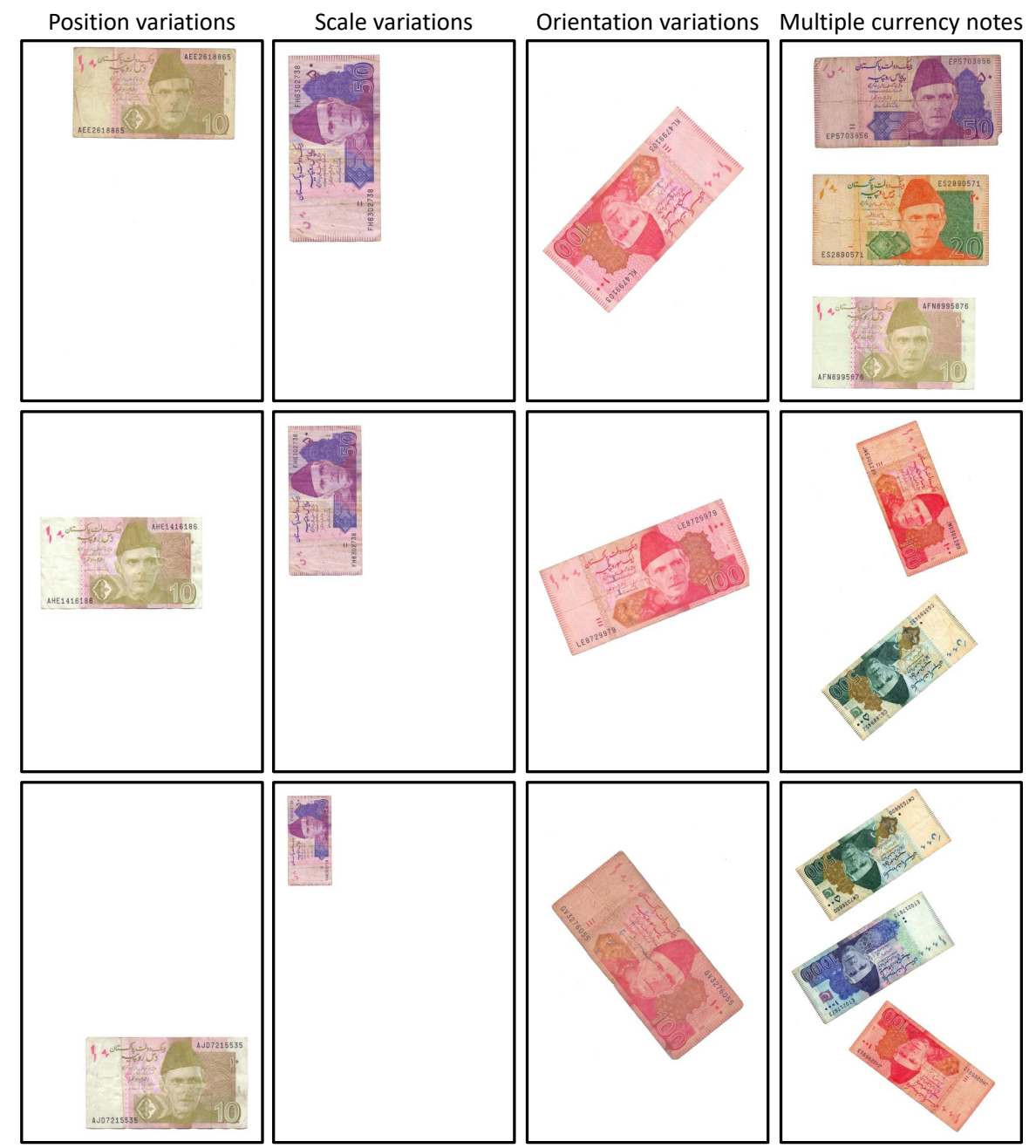

Figure 1. Common image variations found in currency note images.

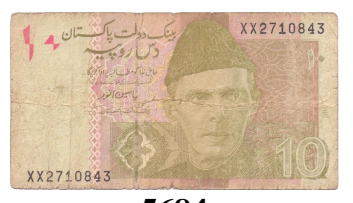

5684

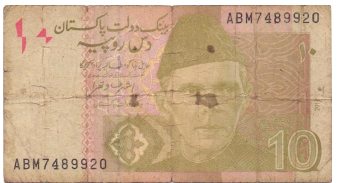

5738

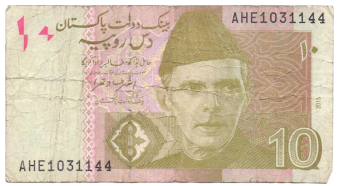

5778

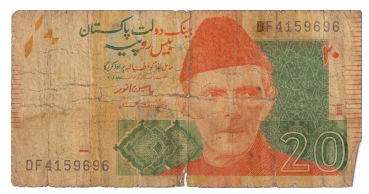

5274

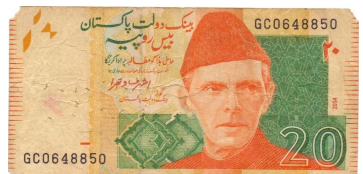

5304

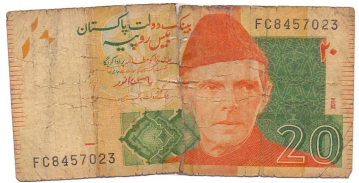

5506

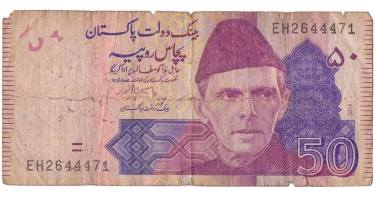

5096

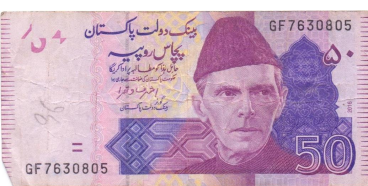

4953

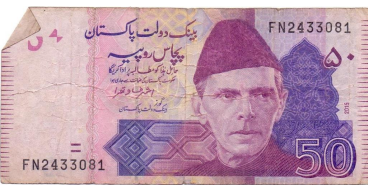

5000

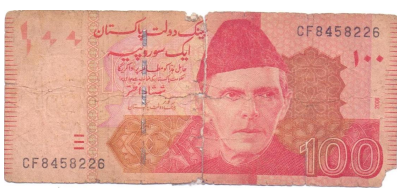

4733

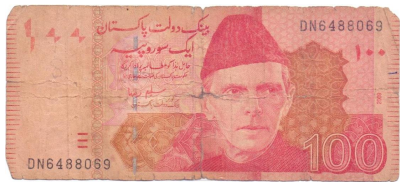

4754

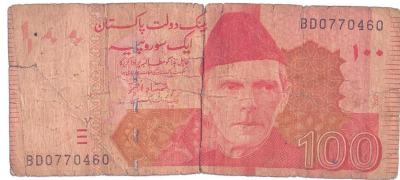

4709

Figure 2. Variations in aspect ratios due to irregular currency note boundaries. The aspect ratio of each currency note is shown below it. 


\subsection{Related Work}

Based on their approaches, the proposed solutions for currency denomination recognition can broadly be divided in the following three classes.

Machine learning-based methods:

The first class of methods uses machine learning techniques such as artificial neural networks (ANN) and support vector machines (SVMs). For instance Takeda and Omatu [4] used a mask of slabs that is convoluted with a local pixel neighborhood such that the coefficients of the masks are randomly chosen to be either 1 or 0 . The aggregated results of several such convolutions are then provided as input to a three layered neural network (NN). Similarly, in Frosini et al. [8], the light refracted from the bank notes was captured with arrays of opto-electronic sensors and then provided to a multi-layered perceptron. Takeda and Nishikagi [9] extracted the information of the currency notes via the so-called axis symmetric masks and then provided it to an ANN. However, more recently, a state-of-the-art performing convolutional neural network (CNN) was used by Pham et al. [10] where they reported a result of $100 \%$ on an image dataset of 64,000 images belonging to 64 classes. In addition to the neural networks and its variants, other machine learning algorithms such SVM were also used along with its various types of kernels. In Chang et al. [11], the features constructed from sensors were used to represent the key regions of a currency note to predict whether it is real or fake. The task of classification is performed by a support vector machine (SVM) where different kernels were evaluated for classification accuracy. Similarly, He et al. [12] used principle component analysis (PCA) features to represent an edge image of a single banknote. A genetic algorithm (GA) was further used for feature selection. Such image representation was then used to train an SVM model, and later for testing it. Other machine learning algorithms used for currency recognition include the hidden Markov model (HMM) [13], k nearest neighbors (kNN) [14] and Gaussian mixture model (GMM) [15].

Local feature matching-based methods:

A local feature descriptor is a compact representation of the pixel intensity distribution within a local image patch. As a common practice, the feature descriptor is an $\mathrm{N}$ dimensional vector. For object recognition via feature matching, the local features such as scale-invariant feature transform (SIFT) [3] are extracted from the representative images of a given object and stored in a database. Then, from a given image, local features are extracted and matched with the ones using a distance measure such as the Euclidean distance. The local features of the stored image that are the nearest to the local features of the test image are declared to belong to the same object class. The local feature matching is also used for currency recognition. SIFT and its color variant also known as color SIFT are used for currency recognition by [16]. Similarly, Hasanuzzaman et al. [1] used speedup robust features (SURF) for currency recognition. More recently, Yousry et al. [17] used binary local feature descriptor oriented FAST and rotated BRIEF (ORB) to represent the input image and then compared with the representative images in the database using the Hamming distance. Other local features used for currency recognition include local binary patterns (LBP) [18] and a gray-level co-occurrence matrix (GLCM) [19].

Image processing-based methods:

The image processing based methods are purely based on pixel level manipulation techniques such as extracting the region of interest (ROI) of a banknote and then applying various measures such as the correlation [20]. Such methods are more relevant for real-time applications where relatively less processing resources are available. Similarly, Youn et al. [5] used the banknote size information along with multiple-template matching for a multiple currency recognition system.

The proposed paper currency recognition method is based on a two step process. The first step deals with the extraction of paper currency note from the image via local entropy and range filters. The extracted note region is then normalized and rotated to achieve scale- , translation-, and 
rotation-invariance. The second step is recognition of the extracted and processed region. This is done by computing the aspect ratio of the extracted region as currency notes differ from one another based on this feature. Since, the aspect ratio of a rectangular object is not affected by the scale, position, and in-plane orientation of the object, it is also invariant to these transformations. Hence, our proposed paper currency recognition method is invariant to changes in scale, position, and in-plane orientations.

\section{Methodology}

The proposed method is inspired by an automatic ancient coin segmentation [21]. Following their method, we also segment a currency note that is imaged on a homogeneous background by the following assumptions.

1. The image region depicting a currency note contains the highest information content.

2. The most rectangular object found in the image is a currency note that has a predefined but slightly varying aspect ratio depending on its condition.

Therefore, the segmentation becomes a two step process. The first step deals with the extraction of image regions with the highest information contents. The second step consists of finding rectangular regions among the extracted ones and calculating their aspect ratios. In the following, we elaborate on both of these steps.

\subsection{Informative Region Extraction}

Since, the currency note is imaged on a homogeneous background, the image region depicting the note is likely to have more variations in terms of pixel values than the background. We employ a local image neighborhood processing strategy to extract informative regions. The local image neighborhood is simply a subset of image pixels arranged in rows and columns. The image filters that process a local image neighborhood are called the local filters. In our proposed method, we use the following two local filters.

Local entropy filter:

Entropy gives the measurement of information content in an event, signal, or in our case, an image. Concretely, entropy is inversely proportional to the probability of a random variable where it is maximum if the value of probability is close to zero and vice versa. In the context of image, the information content is represented by the pixel intensity values that range from 0 to $L-1$ where $L=256$. The histogram of pixel intensity values [22] represents the number of pixels per intensity value as shown in Equation (1).

$$
h(i)=n_{i} \quad i \in 0,1,2, \ldots, 255 .
$$

Each bin in this histogram gives the count of that particular intensity value as $n_{0}, n_{1}, \ldots, n_{(L-1)}$. In other words, the total number of pixels in the local neighborhood having intensity value $i$, is $n_{i}$.

In order to convert this count into their respective probabilities, the histogram of intensity values is normalized by dividing the value of each bin over the total number of pixels in the local neighborhood. For instance, if the total number of rows in a local neighborhood is $M$ and the total number of columns is $N$, then, the normalized histogram is given as,

$$
p(i)=h(i) /(M * N)=n_{i} /(M * N), \quad i \in 0,1,2, \ldots, 255
$$

where, $p(0), p(1), \ldots, p(255)$ give the probabilities of each intensity value in the local neighborhood.

The entropy of a local neighborhood $\Omega$ in an image is then found via this normalized histogram using Equation (3),

$$
H(\Omega)=-\sum_{i=0}^{255} p(i) \cdot \log _{2}(p(i))
$$


Local range filter:

The range of a local neighborhood is simply calculated by finding the difference of maximum intensity value in that particular neighborhood and the minimum.

To summarize, the response of local entropy filter for homogeneous image regions will be minimum whereas it will be maximum for those regions having higher variations of pixel intensity values. A similar result will be achieved for the local range filter. Figure 3 shows the same effect where we depict the responses of both the filters on a one dimensional signal that is a single row of a currency note image. The flat part of the intensity signal corresponds to the homogeneous background while its fluctuating part shows the variations in pixel intensity values of the currency note. Consequently, both the local filters achieve results that are adequate for both the homogeneous and fluctuating part. It can be observed that they do not respond to the homogeneous part while their responses are more pronounced to the part of signal representing the pixel intensity variations.
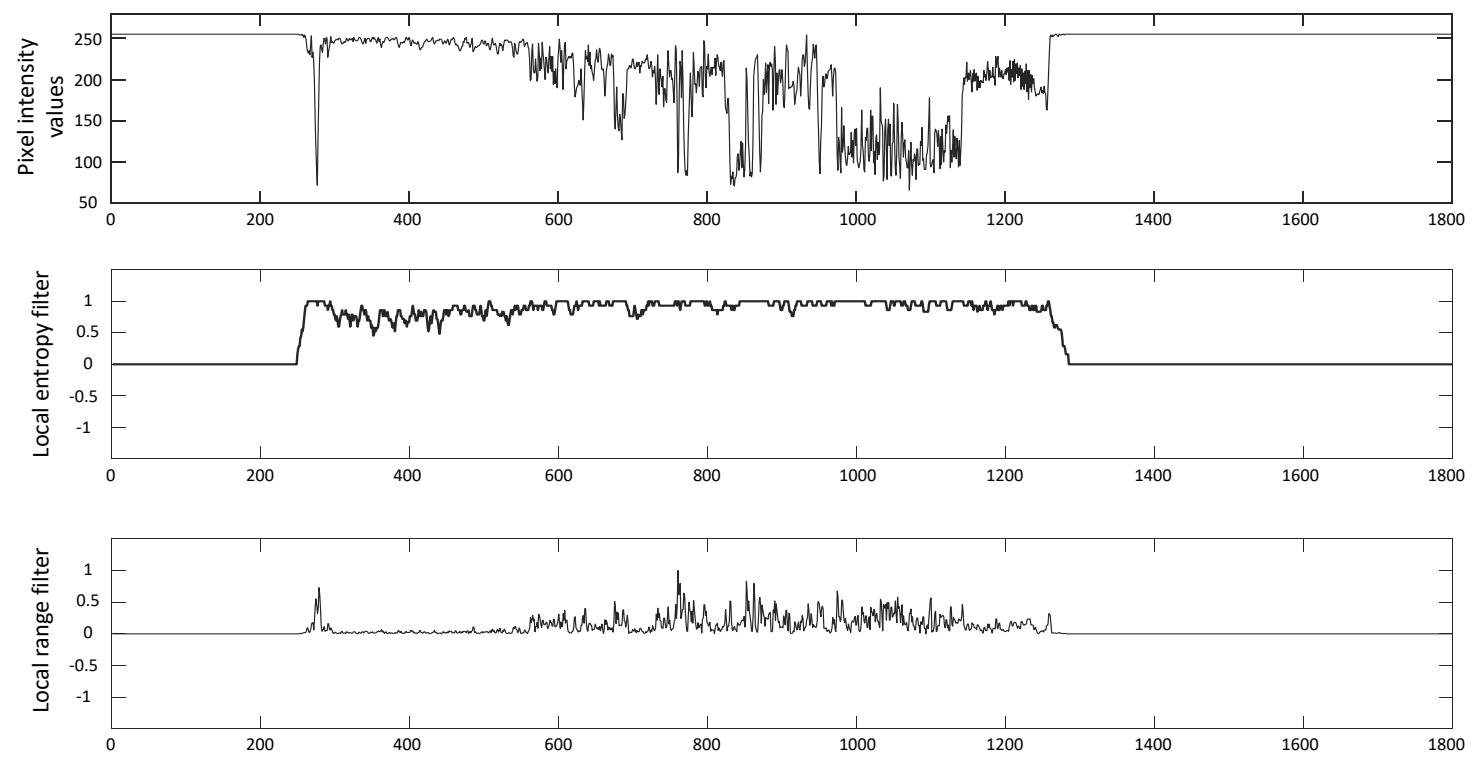

Figure 3. Responses of local entropy and range filters on a single row of currency note images (where the values at $x$-axis show the number of rows).

Sum of both filters:

Both these local filters are applied to a given note image. For each filter, the size of a local circular neighborhood is empirically selected as 3 . The resultant response of each filter is scaled to the range between 0 and 1. Finally, both the responses are summed to get the combined response of both the filters. The individual and cumulative responses of both filters are shown in Figure 4. The region of images where both the filters give high response shows more informativeness than the background. However, the border region of the currency note in the combined response is more pronounced, which proves supportive at the stage of segmentation. 


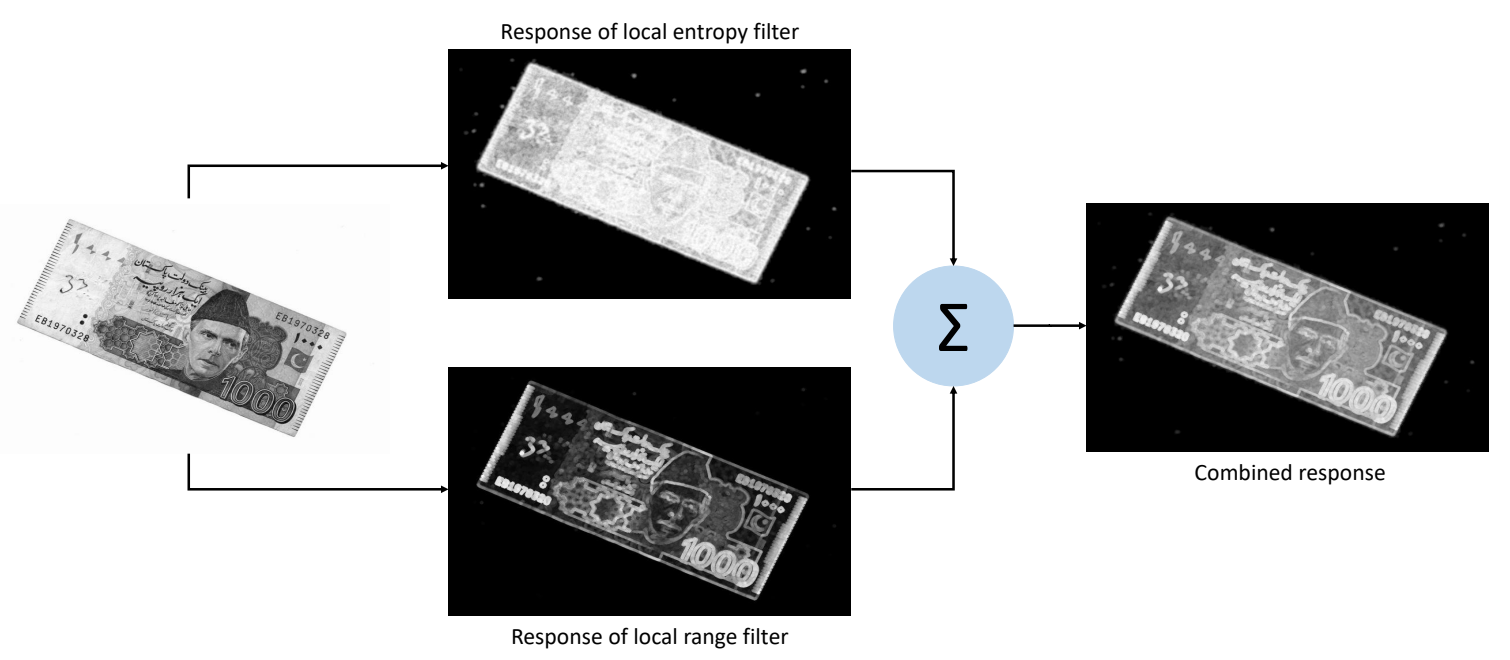

Figure 4. Responses of local entropy and range filters on a currency note image.

\subsection{Currency Note Segmentation and Recognition via Aspect Ratio}

For the current imaging conditions, a set of empirically defined thresholds $(0.3,0.4,0.5$, and 0.6$)$ is applied to the combined response image for segmenting the currency note region. All the binarized images produced by each threshold are summed to get the final segmentation mask for the currency note. This whole procedure is shown in Figure 5. The resulting binarized masks generated by applying each threshold are very similar except for the last one. This effect is more or less observed on all the images of the dataset. As a last step, the aspect ratio of the generated mask is calculated to determine the denomination of currency note. To this end, we evaluated two kinds of aspect ratios for each denomination that are further explained in Section 4.

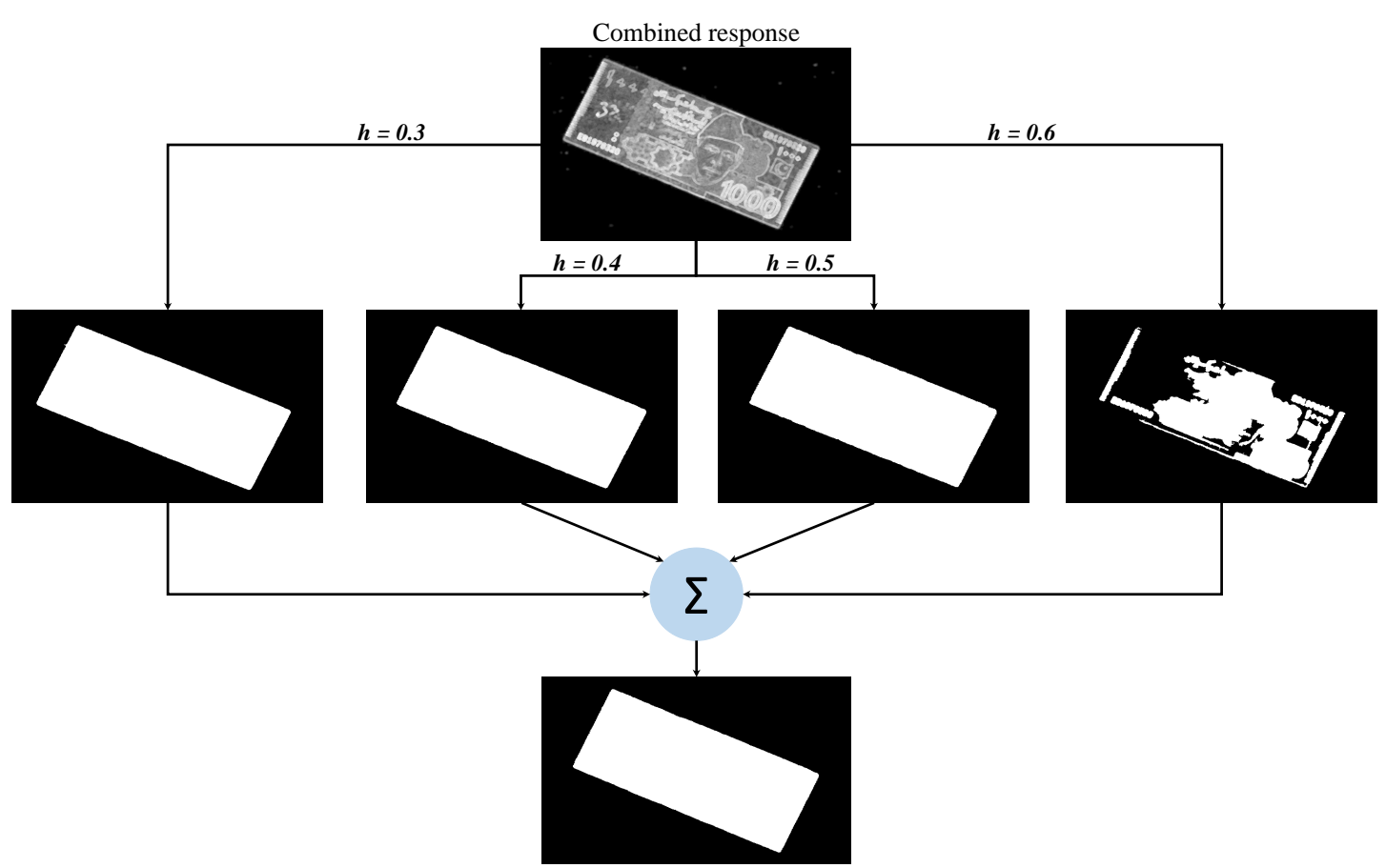

Figure 5. The process of segmentation mask generation using threshold-defined binarization masks.

\section{A New Pakistani Currency Notes Dataset (PCND):}

We collect an image dataset of Pakistani notes with images having high variations with respect to in-plane orientation and position. These images are taken with a desktop flatbed scanner where the background is completely homogeneous. All the seven denominations of the Pakistani Currency of 
the 2005 series are represented in the dataset. We divide our dataset into two disjoint subsets. The first subset that we call the "training set" is used to calibrate the aspect ratio for each note. This is further elaborated in Section 4. We call the second dataset the "test dataset", where currency notes of various denominations are scanned at arbitrary positions and orientations in the following three different settings.

1. This is the simplest setting with only one currency note per image.

2. The images in this setting contain two currency notes of different denominations. However, the notes are separated from each other to such an extent that they are not overlapped. Overlapping will cause their boundaries to merge thus resulting in a wrong segmentation. For this setting, the notes of consecutive denominations are chosen as they are more likely to get confused.

3. Finally, three notes per image of consecutive denominations are imaged together.

It should be noted that the images with multiple notes are simply made by combining the single note images such that they are scaled, rotated and positioned arbitrarily. Table 2 shows the total number of images per denomination in both subsets of the image dataset.

Table 2. Details of image dataset.

\begin{tabular}{cccc}
\hline \multicolumn{4}{c}{$\begin{array}{c}\text { Number of Images per Denomination } \\
\text { Training Set }\end{array}$} \\
Denomination & No. of Images & Denomination & No. of Images \\
\hline 10 & 105 & 10 & 100 \\
20 & 101 & 20 & 97 \\
50 & 101 & 50 & 96 \\
100 & 94 & 100 & 97 \\
500 & 101 & 500 & 77 \\
1000 & 101 & 1000 & 90 \\
5000 & 9 & 5000 & 41 \\
\hline
\end{tabular}

\section{Results and Discussion}

Since the proposed currency note recognition method is based on the aspect ratios of the banknotes, as a first step, the values of aspect ratios for each denomination have to be established. We simply divide the shorter side (width) of the note over its longer side (length) and then scale the number by multiplying it with 10,000. For instance the standard width of the currency note of 10 rupees is $65 \mathrm{~mm}$ and its standard length is $115 \mathrm{~mm}$. Therefore, its aspect ratio is $10,000 \times(65 / 115)$, which is 5652. We adapt the following two different methods to obtain the aspect ratio for each denomination.

1. Standard aspect ratio: This is the aspect ratio of an original and new currency note.

2. Calibrated aspect ratio: The majority of the notes used in the market undergo wear and tear due to their age and usage. These currency notes are not in their original shape and thus their aspect ratios are more likely to differ from the new ones. We use currency note images in the training set to "calibrate" the aspect ratio for each denomination. To this end, we find the aspect ratios of currency notes for each denomination in the training set that include both new and used notes. The mean of all these aspect ratios is then considered as the aspect ratio of the respective denomination.

Both the standard and the calibrated aspect ratios for each denomination are shown in Table 3. The difference between standard and calibrated aspect ratios for each denomination can be observed. This is due to the fact that training set consists of used currency notes whose aspect ratios vary from one another due to the deterioration in their shapes. Once, the aspect ratios are established for each note type, the next step is to use them to label a given test note image. From a given image, the note image is extracted and then its aspect ratio is calculated using the proposed method. This aspect ratio is then compared with the aspect ratios of all the notes and the one nearest to it is assigned the label. 
Table 3. Values of standard and calibrated aspect ratios for different denominations.

\begin{tabular}{lccccccc}
\hline & $\mathbf{1 0}$ & $\mathbf{2 0}$ & $\mathbf{5 0}$ & $\mathbf{1 0 0}$ & $\mathbf{5 0 0}$ & $\mathbf{1 0 0 0}$ & $\mathbf{5 0 0 0}$ \\
\hline Standard aspect ratio & 5652 & 5285 & 4962 & 4676 & 4422 & 4194 & 3988 \\
Calibrated aspect ratio & 5750 & 5356 & 5055 & 4762 & 4526 & 4295 & 4083 \\
Difference & 98 & 71 & 93 & 86 & 104 & 101 & 95 \\
\hline
\end{tabular}

Table 4. Denomination recognition rates achieved on standard and calibrated aspect ratios where the recognition rates of calibrated aspect ratios (in bold) are better than those of the standard aspect ratios

\begin{tabular}{lcccccccc}
\hline & $\mathbf{1 0}$ & $\mathbf{2 0}$ & $\mathbf{5 0}$ & $\mathbf{1 0 0}$ & $\mathbf{5 0 0}$ & $\mathbf{1 0 0 0}$ & $\mathbf{5 0 0 0}$ & Overall \\
\hline Standard aspect ratio & $100 \%$ & $97 \%$ & $96 \%$ & $97 \%$ & $77 \%$ & $90 \%$ & $41 \%$ & $89 \%$ \\
Calibrated aspect ratio & $100 \%$ & $\mathbf{9 9 \%}$ & $\mathbf{9 8 \%}$ & $\mathbf{9 9 \%}$ & $\mathbf{1 0 0 \%}$ & $\mathbf{9 9 \%}$ & $\mathbf{1 0 0} \%$ & $\mathbf{9 9 \%}$ \\
\hline
\end{tabular}

The images per note type vary from 60 to 100, for a total of 598 images. In each test image, there is a single instance of a note that is displayed at an arbitrary scale, position, and orientation. The results for both the methods are shown in Table 4 while the confusion matrices for each note type are shown in Figure 6. The aspect ratios based on calibration clearly outperform the standard aspect ratios. This is quite realistic as the notes in the test image data contain both new and old notes. Due to this reason, the calibration-based aspect ratios give flexible values for both new and old notes to be recognized. The denomination recognition results for currency notes images with different variation are shown in Figure 7. Our method successfully recognizes denominations of each currency note under the translation, scale, and rotation variations. It also very accurately recognizes the denomination of multiple currency notes that are imaged together. However, a failure on the currency note of 500 can be observed where it is wrongly recognized as a note with the denomination of 100 .
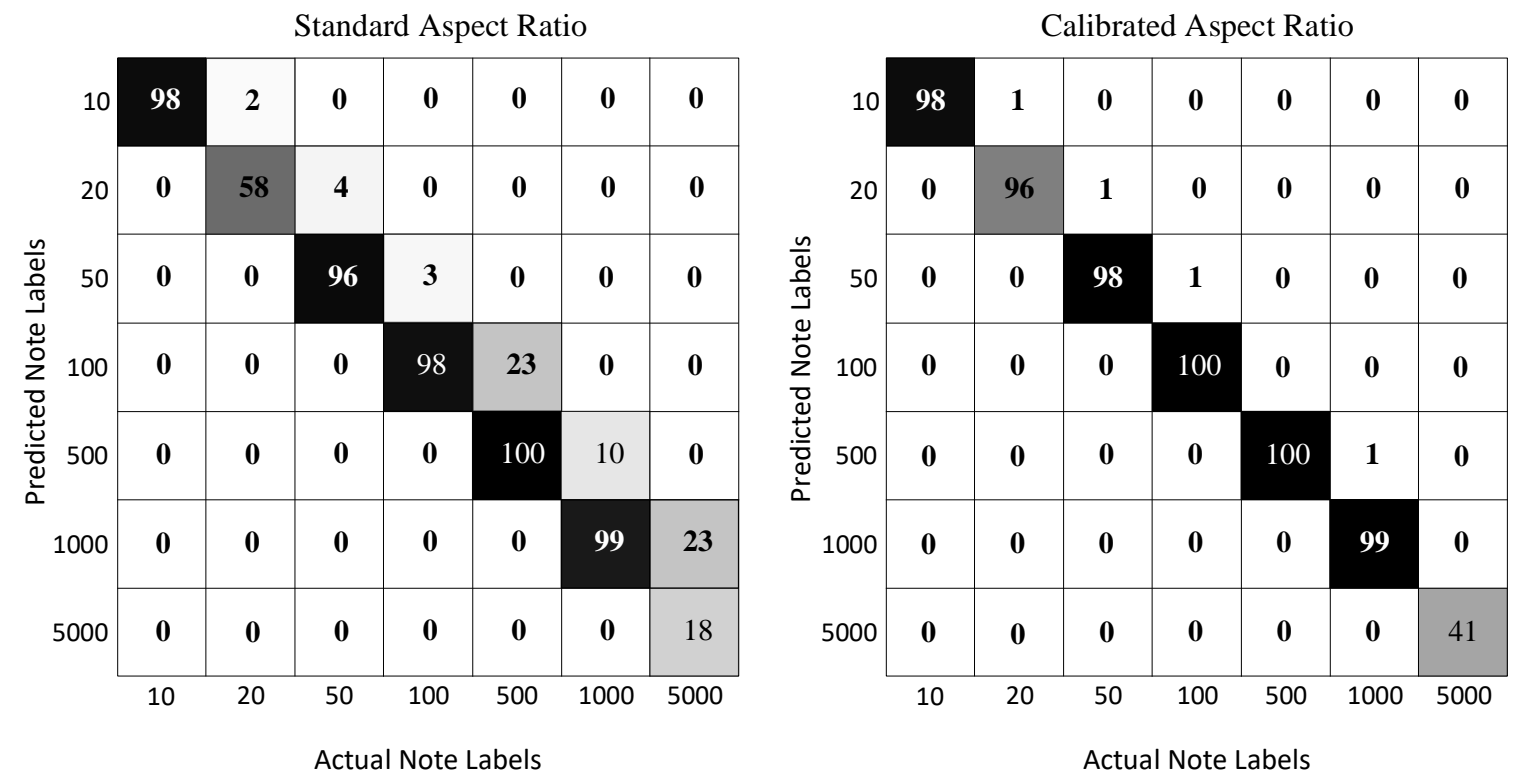

Figure 6. Confusion matrices for both the standard and calibrated aspect ratios. 

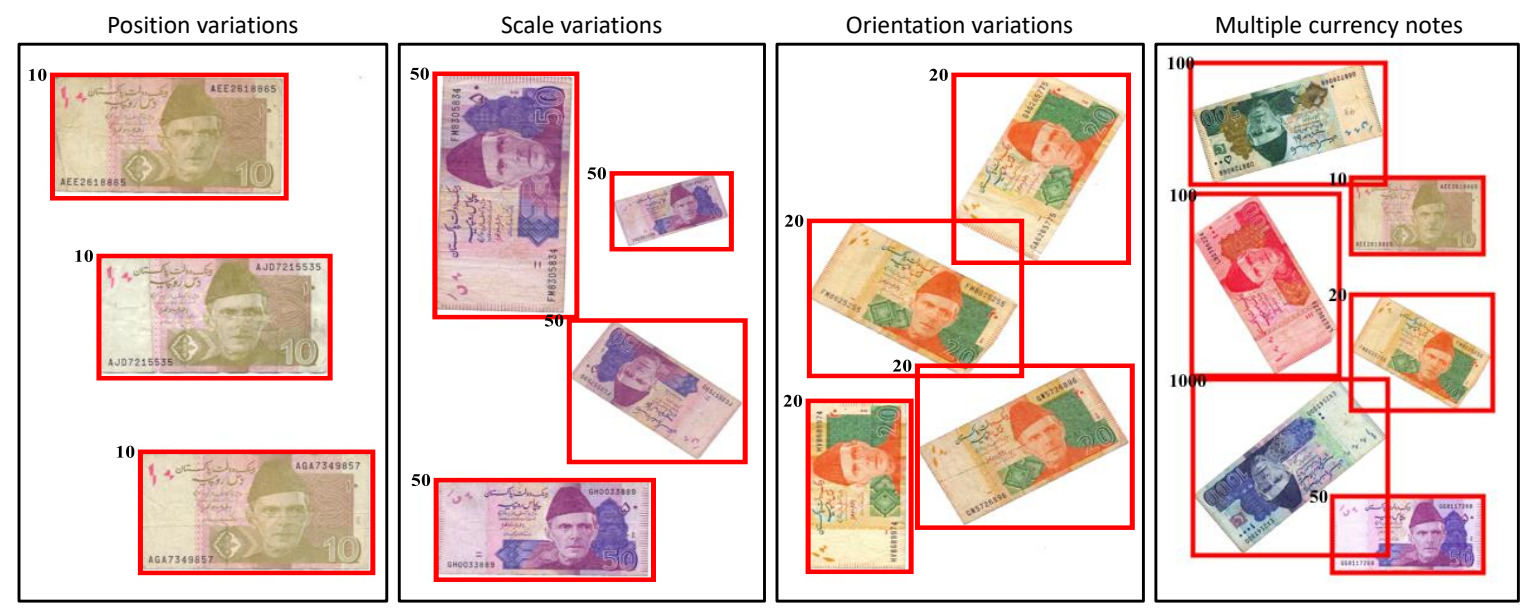

Figure 7. Visualization of denomination recognition of currency notes imaged at various positions, scales and orientations.

\section{Conclusions and Future Work}

We performed denomination recognition of Pakistani currency notes from their images. Such recognition was achieved despite the image variations that are commonly found in currency images. These image variations are mainly caused by changes in currency note position, scale, and orientation. To achieve a denomination recognition that is invariant to these image variations, a two step process was proposed where, in the first step, informative regions from the images are extracted via local entropy and range filters. As a second step, these regions are extracted via binarization and their aspect ratios are calculated for final denomination recognition. The aspect ratio for each denomination is established by using a training set where, for each denomination, notes are used with various degrees of deterioration based on their usage. The proposed method is evaluated on a novel dataset of Pakistani currency notes of the 2005 series, where it achieves a recognition rate of $99 \%$ on a total of 598 images. The usage of a single feature, i.e. aspect ratio, for recognition makes our method more feasible for real-time application which we plan to test in the future by implementing this method on a Raspberry Pi.

Author Contributions: Methodology, H.A.; Validation, F.U.; Conceptualization, A.I.; Data curation, A.U.H.; Conceptualization, A.U.R.; Editing and funding acquisition, P.B.; Methodology, writing-review and editing, D.K.

Funding: We acknowledge support by Deutsche Forschungsgemeinschaft and Friedrich-Alexander-Universität Erlangen-Nürnberg (FAU) within the funding program Open Access Publishing. The research was also partially funded by the Untenured Faculty Research Initiative (UFRI), Kean University.

Conflicts of Interest: The authors declare no conflict of interest.

\section{References}

1. Hasanuzzaman, F.M.; Yang, X.; Tian, Y Robust and Effective Component-Based Banknote Recognition for the Blind. IEEE Trans. Syst. Man Cybern. Part C (Appl. Rev.) 2012, 42, 1021-1030. doi:10.1109/TSMCC.2011.2178120. [CrossRef] [PubMed]

2. Sargano, A.B.; Sarfraz, M.; Haq, N. Robust features and paper currency recognition system. In Proceedings of the 6th International Conference on Information Technology (ICIT 2013), Amman, Jordan, 8-10 May 2013; pp. $8-10$.

3. Lowe, D.G. Distinctive Image Features from Scale-Invariant Keypoints. Int. J. Comput. Vision 2004, 60, 91-110. doi:10.1023/B:VISI.0000029664.99615.94. [CrossRef]

4. Takeda, F.; Omatu, S. High Speed Paper Currency Recognition by Neural Networks. Trans. Neur. Netw. 1995, 6, 73-77. doi:10.1109/72.363448. [CrossRef] [PubMed]

5. Youn, S.; Choi, E.; Baek, Y.; Lee, C. Efficient multi-currency classification of CIS banknotes. Neurocomputing 2015, 156. doi:10.1016/j.neucom.2015.01.014. [CrossRef] 
6. Anwar, H.; Zambanini, S.; Kampel, M. A Bag of Visual Words Approach for Symbols-Based Coarse-Grained Ancient Coin Classification. arXiv 2013, arXiv:1304.6192. Available online: https:/ /arxiv.org/abs/1304.6192 (accessed on 30 October 2019).

7. Anwar, H.; Zambanini, S.; Kampel, M. Encoding Spatial Arrangements of Visual Words for RotationInvariant Image Classification. In Pattern Recognition; Jiang, X., Hornegger, J., Koch, R., Eds.; Springer International Publishing: Cham, Germany, 2014; pp. 443-452.

8. Frosini, A.; Gori, M.; Priami, P. A neural network-based model for paper currency recognition and verification. IEEE Trans. Neural Networks 1996, 7, 1482-1490. doi:10.1109/72.548175. [CrossRef] [PubMed]

9. Takeda, F.; Nishikage, T. Multiple Kinds of Paper Currency Recognition Using Neural Network and Application for Euro Currency. In Proceedings of the IEEE-INNS-ENNS International Joint Conference on Neural Networks. IJCNN 2000. Neural Computing: New Challenges and Perspectives for the New Millennium, Como, Italy, 27 July 2000; IEEE Computer Society: Washington, DC, USA, 2000. IJCNN '00. pp. 143-147.

10. Pham, T.; Eun Lee, D.; Ryoung Park, K. Multi-National Banknote Classification Based on Visible-light Line Sensor and Convolutional Neural Network. Sensors 2017, 17, 1595. doi:10.3390/s17071595. [CrossRef] [PubMed]

11. Chen Chang, C.; Xing Yu, T.; Yen Yen, H. Paper Currency Verification with Support Vector Machines. In Proceedings of the 2007 Third International IEEE Conference on Signal-Image Technologies and Internet-Based System, Shanghai, China, 16-18 December 2008; pp. 860-865. doi:10.1109/SITIS.2007.146. [CrossRef]

12. He, J.B.; Zhang, H.M.; Liang, J.; Jin, O.; Li, X. Paper Currency Denomination Recognition Based on GA and SVM. Paper Currency Denomination Recognition Based on GA and SVM. In Proceedings of the Chinese Conference on Image and Graphics Technologies, Beijing, China, 19-20 June 2015; pp. 366-374.

13. Hassanpour, H.; Farahabadi, P.M. Using Hidden Markov Models for Paper Currency Recognition. Expert Syst. Appl. 2009, 36, 10105-10111. doi:10.1016/j.eswa.2009.01.057. [CrossRef]

14. HLAING, K.N.N. First order statistics and GLCM based feature extraction for recognition of Myanmar paper currency. In Proceedings of the IIER International Conference, Bangkok Thailand, 17 June 2015; pp. 1-6.

15. Jin, Y.; Song, L.; Tang, X.; Du, M. A Hierarchical Approach for Banknote Image Processing Using Homogeneity and FFD Model. IEEE Signal Process. Lett. 2008, 15, 425-428. [CrossRef]

16. Doush, I.A.; Sahar, A.B. Currency recognition using a smartphone: Comparison between color SIFT and gray scale SIFT algorithms. J. King Saud Univ. Comput. Inf. Sci. 2017, 29, 484-492.

17. Yousry, A.; Taha, M.; Selim, M. Currency Recognition System for Blind people using ORB Algorithm. Int. Arab J. Inf. Technol. 2018, 5, 34-40.

18. Sharma, B.; Kaur, A. Recognition of Indian paper currency based on LBP. Int. J. Comput. Appl. 2012, 59, $24-27$. doi:10.5120/9514-3913. [CrossRef]

19. Yan, W.Q.; Chambers, J.; Garhwal, A. An empirical approach for currency identification. Multimedia Tools Appl. 2015, 74, 4723-4733. [CrossRef]

20. Semary, N.; Fadl, S.; Eissa, M.; Gad, A. Currency Recognition System for Visually Impaired: Egyptian Banknote as a Study Case. In Proceedings of the 5th International Conference on Information \& Communication Technology and Accessibility, Marrakesh, Morocco, 21-23 December 2015; pp. 1-6.

21. Zambanini, S.; Kampel, M. Robust Automatic Segmentation of Ancient Coins. In Proceedings of the VISAPP, Liboa, Portugal, 5-8 February 2009; pp. 273-276.

22. Ullah, F.; Anwar, H.; Shahzadi, I.; Ur Rehman, A.; Mehmood, S.; Niaz, S.; Mahmood Awan, K.; Khan, A.; Kwak, D. Barrier Access Control Using Sensors Platform and Vehicle License Plate Characters Recognition. Sensors 2019, 19, 3015. [CrossRef] [PubMed]

(C) 2019 by the authors. Licensee MDPI, Basel, Switzerland. This article is an open access article distributed under the terms and conditions of the Creative Commons Attribution (CC BY) license (http:/ / creativecommons.org/licenses/by/4.0/). 